Relations industrielles

Industrial Relations

\title{
Hélène David : Femmes et emploi : le défi de l'égalité. Montréal, Les Presses de l'Université du Québec, 1986, 477 pp., ISBN 2-7605-0393-3
}

\section{Carla Lipsig}

\section{Volume 45, numéro 1, 1990}

URI : https://id.erudit.org/iderudit/050570ar

DOI : https://doi.org/10.7202/050570ar

Aller au sommaire du numéro

\section{Éditeur(s)}

Département des relations industrielles de l'Université Laval

ISSN

0034-379X (imprimé)

1703-8138 (numérique)

Découvrir la revue

Citer ce compte rendu

Lipsig, C. (1990). Compte rendu de [Hélène David : Femmes et emploi : le défi de l'égalité. Montréal, Les Presses de l'Université du Québec, 1986, 477 pp., ISBN 2-7605-0393-3]. Relations industrielles / Industrial Relations, 45(1), 202-203. https://doi.org/10.7202/050570ar

Tous droits réservés @ Département des relations industrielles de l'Université Laval, 1990
Ce document est protégé par la loi sur le droit d'auteur. L'utilisation des services d'Érudit (y compris la reproduction) est assujettie à sa politique d'utilisation que vous pouvez consulter en ligne.

https://apropos.erudit.org/fr/usagers/politique-dutilisation/ 


\section{RECENSIONS BOOK REVIEWS}

Femmes et emploi: le défi de l'égalité, par Hélène David, Montréal, Les Presses de l'Université du Québec, 1986, 477 pp., ISBN 2-7605-0393-3

Dans son livre récent intitulé Femmes et emploi: Le défi de l'égalité, Hélène David, chercheuse à l'Institut de recherche appliquée sur le travail, esquisse les origines de la discrimination à l'égard des femmes au travail et offre un bilan et une analyse des mesures législatives visant à la redresser. L'ouvrage est important - le premier et le seul à traiter de ce sujet au Québec. Doué d'un élégant schéma d'analyse, rempli d'informations et de cas tirés de nombreux pays et bien intégrés à la situation québécoise, le livre est une mine d'or.

Le point de départ de l'ouvrage repose sur deux constats: premièrement, que la discrimination à l'égard des femmes au travail est un phénomène systémique, c'est-à-dire un ensemble d'éléments qui structurent le marché du travail, dressant des obstacles institutionnels puissants contre l'accès à l'égalité; deuxièmement, pour contrer la discrimination systémique, il faut une stratégie de riposte systémique.

Dans la première partie du livre, l'auteure dresse un bilan de la discrimination - «un héritage du passé" - à l'égard des femmes au travail, commençant par l'inégalité salariale, passant par la ségrégation sexuelle du marché du travail et les politiques discriminatoires dans l'embauche, l'affectation, la mobilité et la formation professionnelle. Dans la deuxième partie de l'ouvrage (la plus intéressante), on analyse les approches législatives utilisées par plusieurs pays pour redresser la discrimination. Se basant sur les Etats-Unis, l'auteure prétend qu'il y a trois étapes historiques dans la conceptualisation socio-législative de la discrimination: débutant par la notion de la discrimination intentionnelle, passant par la discrimination ouverte, pour aboutir, aujourd'hui, à la notion de discrimination systémique. Elle distingue entre les stratégies législatives françaises et suédoises d'un côté et les approches américaines, canadiennes et québécoises de l'autre.

L'analyse longue et fascinante du rapport conceptuel entre les lois américaines originales contre la discrimination raciale et l'orientation actuelle de la législation à l'égard de l'égalité des femmes se justifie par les liens que l'auteure réussit à tracer entre ce cheminement américain et la situation québécoise. Pour leur part, les deux chapitres sur la législation antidiscriminatoire au Canada et au Québec offrent à la fois un aperçu des débats qui ont entouré l'adoption de ces lois et une analyse percutante de leurs limites.

Somme toute, l'ouvrage répond bien à un de ses objectifs: évaluer l'efficacité des moyens juridiques de combattre la discrimination. Un ouvrage solide, accessible, plein de cas concrets et à la fine pointe de certains problèmes futurs. Pourquoi, donc, sommes-nous laissés sur nos appétits?

Pour deux raisons: premièrement, étant donné l'importance accordée à l'analyse globale des sources de la discrimination et de l'efficacité des mesures de redressement, on aurait souhaité une discussion de l'évolution historique du rôle des femmes dans le marché du travail 
capitaliste et de l'évolution historique des politiques de l'Etat envers "l'insertion" des femmes dans le marché du travail; deuxièmement, si la discrimination systémique exige une stratégie de riposte systémique, de toute évidence elle mérite aussi d'être insérée dans le contexte de la lutte actuelle de l'ensemble des travailleurs.

Carla LIPSIG

Université Laval

Discrimination sur le marché du travail et information imparfaite, par Jean-Michel Plassard, Editions du CNRS, France, 1987, 386 pp., ISBN 2-222-04077-9

La discrimination au travail est un phénomène dont l'ampleur et la persistence, malgré les législations anti-discriminatoires en vigueur, ont alimenté et continuent d'alimenter la littérature. Depuis quelques années, les publications portent surtout sur les solutions envisagées pour éliminer toute forme de discrimination au travail. On fait ainsi écho aux interventions concrètes qui prennent place de plus en plus dans les entreprises, sous la forme des programmes d'accès à l'égalité ou de l'équité salariale.

L'ouvrage de Jean-Michel Plassard se situe par contre à un autre niveau puisqu'il s'agit d'un ouvrage théorique, présenté de manière très «académique» sur la théorie de la discrimination. L'auteur se restreint à l'analyse de la discrimination sur le marché du travail, laissant de côté la discrimination qui survient avant l'accès au marché.

L'essentiel de l'ouvrage porte sur la discrimination statistique qui relève de la théorie de l'information. L'auteur a cependant cru bon de présenter d'abord (chapitre I) les différents concepts utilisés par l'approche économique de la discrimination ainsi que les apports et les limites des modèles qui «ont précédé le développement de la théorie statistique de la discrimination» (p. 8). Il présente ensuite les concepts fondamentaux liés à la discrimination statistique (chapitre II). La base de la discrimination statistique se retrouve dans la notion d'incertitude: l'information associée aux transactions entre l'offre et la demande de travail n'est pas parfaite. Selon l'auteur, «le modèle de discrimination statistique constitue un des outils proposés par la théorie pour rendre compte du comportement des employeurs confrontés à une incertitude sur la qualité du travail» (p. 113). Dans cette approche, le processus d'embauche tient ainsi une place centrale dans l'analyse.

Les deux premiers chapitres sont intéressants car on y retrouve une bonne présentation des différents modèles explicatifs de la discrimination. Toutefois on peut déplorer et même questionner, à certains endroits, l'utilisation de termes différents pour référer à la même réalité, ce qui finit par déranger le lecteur et peut porter à confusion. On y parle ainsi, à titre d'exemple, de discrimination pré-marché et de pré-discrimination.

Le chapitre III présente par la suite une première série de modèles qui utilisent l'hypothèse de la discrimination statistique dans le domaine de la discrimination raciale et sexuelle. Les trois derniers chapitres présentent la contribution de l'auteur au modèle de discrimination statistique. Plus particulièrement, l'auteur formalise l'aspect non-intentionnel du processus de discrimination lors de l'embauche.

Ce modèle se démarque ainsi des modèles précédents qui ne pouvaient rendre compte que de la discrimination intentionnelle. Il reste cependant que les praticiens et praticiennes qui interviennent dans les milieux de travail ainsi que les législateurs reconnaissent depuis longtemps cet aspect important du processus discriminatoire. Enfin, la conclusion demeure très générale et très théorique. 